\title{
Ondulation de l'interface métal/oxyde dans le cas des alliages Ti-Zr
}

\author{
I. Demoulin, D. Giosmak et M. Lallemant
}

Laboratoire de Recherches sur la Réactivité des Solides, UA 23 CNRS, Faculté des Sciences Mirande, BP 138, 21004 Dijon cedex, France

\begin{abstract}
Résumé. - L'oxydation de l'alliage Ti-Zr contenant $47,5 \%$ atomique de zirconium sous 100 mbar d'air, à $720^{\circ} \mathrm{C}$ permet l'observation de l'ondulation de l'interface alliage/oxyde. Cette ondulation est interprétée comme la réponse aux contraintes du substrat formé de deux phases cristallographiques ( $\alpha$ et $\beta$ ) de duretés différentes.
\end{abstract}

\begin{abstract}
Oxidation of Ti- $\mathrm{Zr}$ alloys containing 47.5 atomic $\%$ zirconium, at $720^{\circ} \mathrm{C}$ and below 100 mbar of air, allows us to show the undulation of alloy-oxide interface. These undulations appear only for particular conditions of temperature and thickness. This process (interface undulation) could be the "visual" translation of the stresses accomodation. These stresses are resorbed by two crystallographic phases $(\alpha$ and $\beta$ ) exhibiting different mechanical properties.
\end{abstract}

\section{Introduction.}

Létude de l'oxydation des alliages Ti-Zr contenant jusqu'à $28 \%$ atomique de zirconium a permis de mettre en évidence l'intervention de phénomènes chimiques, mécaniques et thermiques au cours des réactions $[1,2]$. Ces processus, couplés ou non, ont pu être reliés à la composition de l'alliage et à la température d'oxydation. La figure 1 résume l'existence dans l'échantillon de ces différents processus.

Létude d'alliages contenant 38,5 - 47,5 - 66,8 et $82 \%$ de zirconium a montré l'existence d'un phénomène particulier : l'ondulation de l'interface métal/oxyde. Il paraissaît donc intéressant de déterminer les conditions d'observation (température, épaisseur, \% atomique en zirconium) de ce phénomène et d'en rechercher les causes et l'origine.

Ce phénomène, très marqué dans le cas de l'oxydation de l'alliage Ti-Zr $(47,5 \%$ at. de zirconium), a fait l'objet d'une étude plus détaillée, présentée dans ce qui suit.

\section{Conditions expérimentales.}

L'alliage Ti-Zr a été fourni par la Société Cezus.

Les études sont réalisées sur des échantillons rectangulaires $(7,5 \times 5 \mathrm{~mm})$, d'épaisseur variant de 0,1 à $3 \mathrm{~mm}$, polis jusqu'au grain 1200, lavés à l'acétone et rincés à l'alcool. 


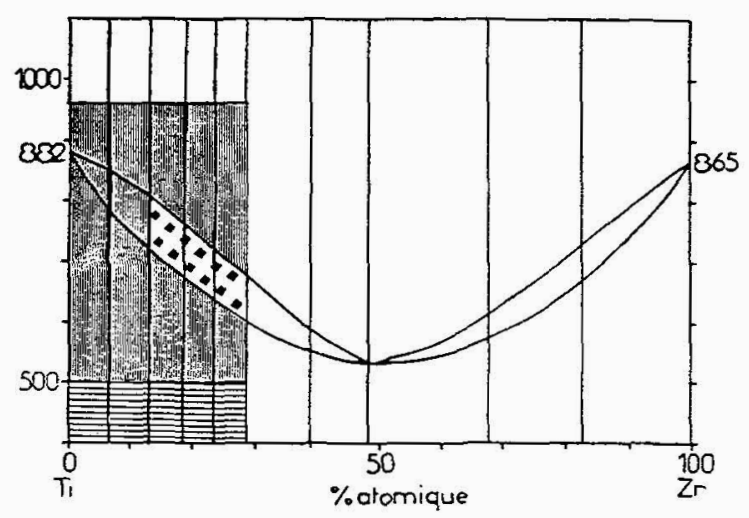

Fig. 1. - Domaines d'existence des différents processus dans l'échantillon : 四 processus chimiques et mécaniques; 舄 processus chimiques; Đprocessus chimiques, mécaniques et thermiques.

L'étude microstructurale réalisée sur la surface de l'échantillon révèle l'existence de grains d'environ $50 \mu \mathrm{m}$ (Photo 1).

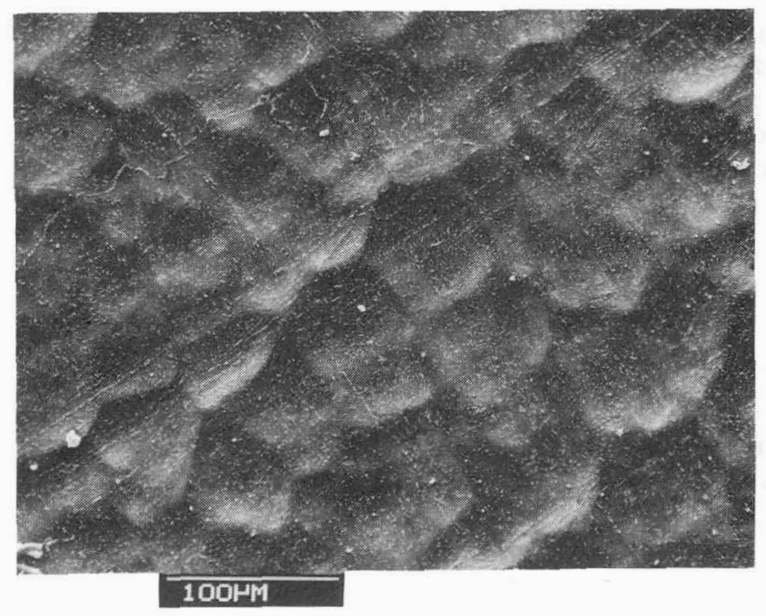

Photo 1. - Microstructure de l'alliage.

L'oxydation a été particulièrement étudiée à $720^{\circ} \mathrm{C}$, sous une pression de $100 \mathrm{mbar}$ d'air et jusqu'à un avancement 0,5 . En effet, ce sont les conditions pour lesquelles les ondulations sont le mieux définies.

\section{Résultats.}

- Pour les échantillons fins ( $e \leq 0,3 \mathrm{~mm}$ ), la surface de l'oxyde est lisse et l'échantillon se déforme ; l'interface alliage/oxyde reste plane (Photo 2a).

- Pour les échantillons plus épais $(0,5 \leq e \leq 1,4 \mathrm{~mm})$, la surface de l'oxyde est squameuse et l'échantillon reste plan. L'interface présente par contre des ondulations assez régulières dont l'amplitude croît avec l'épaisseur de la plaque initiale (Photo 2b). De plus, comme on peut le constater, la morphologie des deux interfaces opposées n'est pas nécessairement la 
même ; en effet, une interface peut présenter une ondulation sans que cette dernière existe nécessairement en vis-à-vis sur l'autre interface.

- Pour les échantillons très épais ( $e=3 \mathrm{~mm}$ ), l'aspect macroscopique devient particulier ; en effet, la croissance de l'oxyde se produit sur les six faces de la plaquette initiale. Par contre, comme pour les faibles épaisseurs, on retrouve une interface plane (Photo 2c).

a

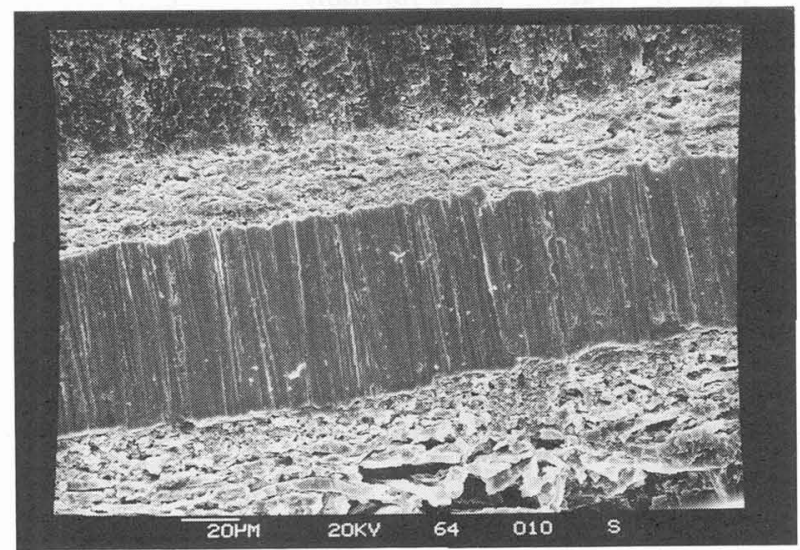

b
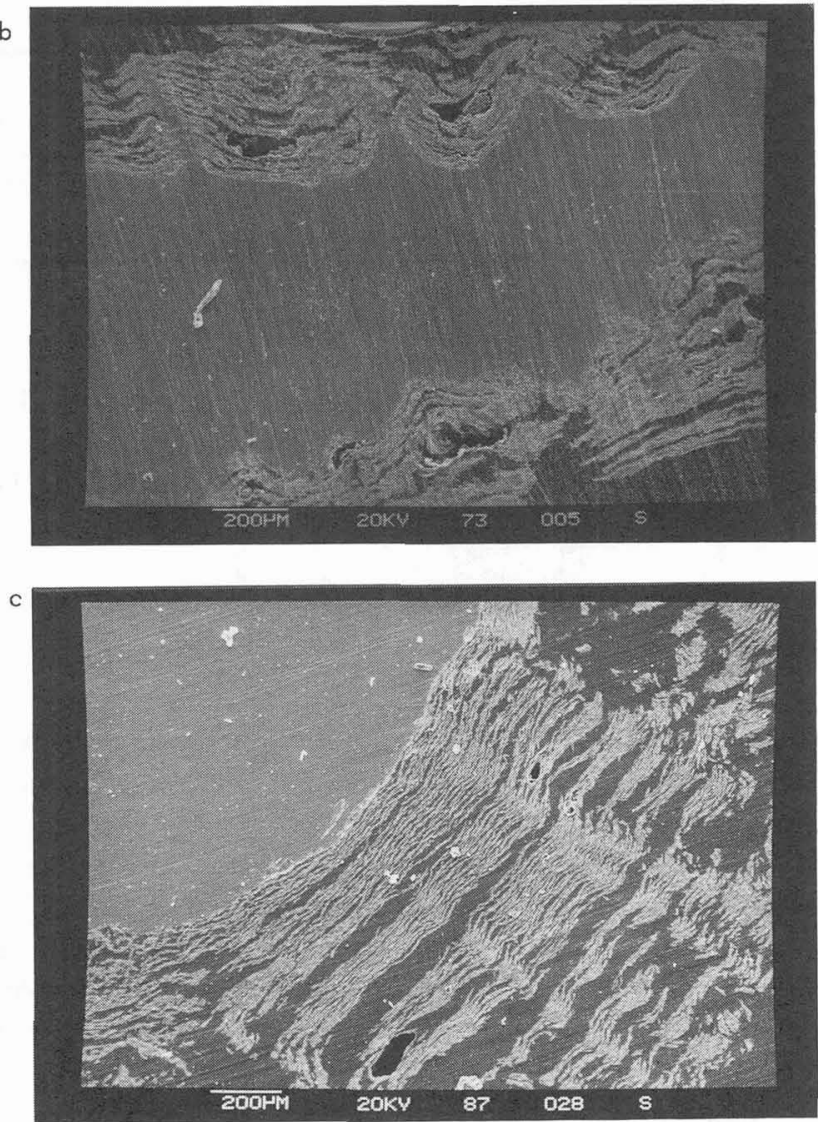

Photo 2. - Interface alliage/oxyde d'un échantillon oxydé à $720^{\circ} \mathrm{C}$ sous 100 mbar d'air $(\xi=0,5)$ : 
On peut résumer les caractéristiques des ondulations de l'interface en fonction de l'épaisseur de la plaque initiale (Tab. I) ; il est à noter que les distances inter-crêtes ne présentent pas une évolution systématique en fonction de l'épaisseur $e$.

Tableau I. - Caractéristiques de l'ondulation en fonction de l'épaisseur de la plaquette initiale e.

\begin{tabular}{lll}
\hline e (mm) & $\begin{array}{l}\text { amplitude de } \\
\text { l'ondulation } \mu \mathrm{m}\end{array}$ & distance inter-crête \\
\hline$\leq 0,3$ & pas d'ondulation \\
\hline 0,5 & 50 & 420 \\
\hline 0,78 & 50 & 320 \\
\hline 1,14 & 90 \\
\hline 1,4 & 450 \\
\hline 3 & \\
\hline
\end{tabular}

La température d'oxydation joue également un rôle prépondérant dans l'apparition des ondulations. A basse température $\left(T \leq 550^{\circ} \mathrm{C}\right)$, l'interface reste plane, comme à haute température $\left(T=800^{\circ} \mathrm{C}\right)$ où l'on observe cependant une rupture de l'oxyde avec étirage des arêtes.

\section{Analyses des résultats.}

Des profils de concentration de titane, zirconium et oxygène (sur les échantillons présentant des ondulations) ont été réalisés par microsonde électronique, en collaboration avec le Laboratoire de Chimie Minérale de l'Université de Nancy, de façon à mettre en évidence d'éventuels processus comme par exemple la ségrégation d'un élément au niveau de l'interface, la diffusion préférentielle aux joints de grains etc...[3].

Cette étude a été réalisée sur la coupe transversale d'un échantillon d'épaisseur initiale $1,14 \mathrm{~mm}$, oxydé jusqu'à l'avancement 0,5 à $720^{\circ} \mathrm{C}$ et présentant des ondulations de forte amplitude. Lanalyse a porté sur une zone étroite A de $84 \mu \mathrm{m}$ d'épaisseur et sur une zone large B de $511 \mu \mathrm{m}$ d'épaisseur (Fig. 2).

Lors de l'observation, des indentations ont été mises en évidence au centre des zones larges (Fig. 2, zone B2), alors que les zones étroites A et les bandes B 1 en étaient exemptes. Ces 


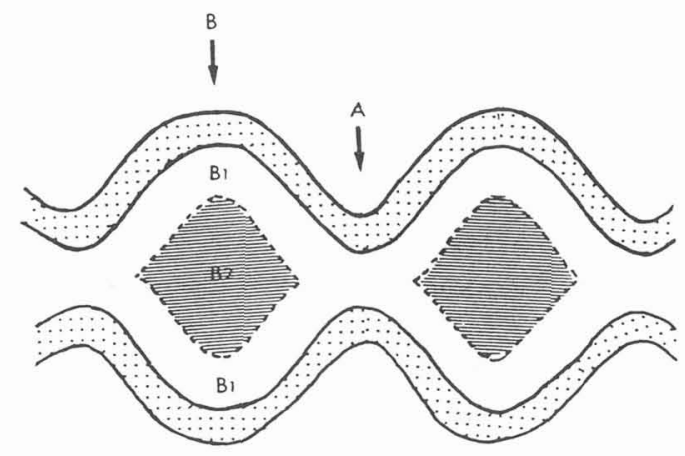

Fig. 2. - Schéma localisant les domaines de dureté différente: 图 oxyde; $\square$ grande dureté (zone $\alpha$ ); 目 faible dureté (zone $\beta$ ).

indentations sont en fait des particules de diamant s'incrustant dans les parties les plus tendres de l'échantillon lors du polissage à la pâte diamantée.

De façon à pouvoir comparer les profils de concentration des zones $\mathrm{A}$ et $\mathrm{B}$, chaque épaisseur (84 et $511 \mu \mathrm{m}$ ) a été ramenée à 100. Les résultats de ces analyses sont résumées figure 3 (pour chaque zone $\mathrm{A}$ ou $\mathrm{B}$, la somme du pourcentage atomique de titane, zirconium et oxygène est égale à 100). L'étude de ce graphique permet deux constatations :

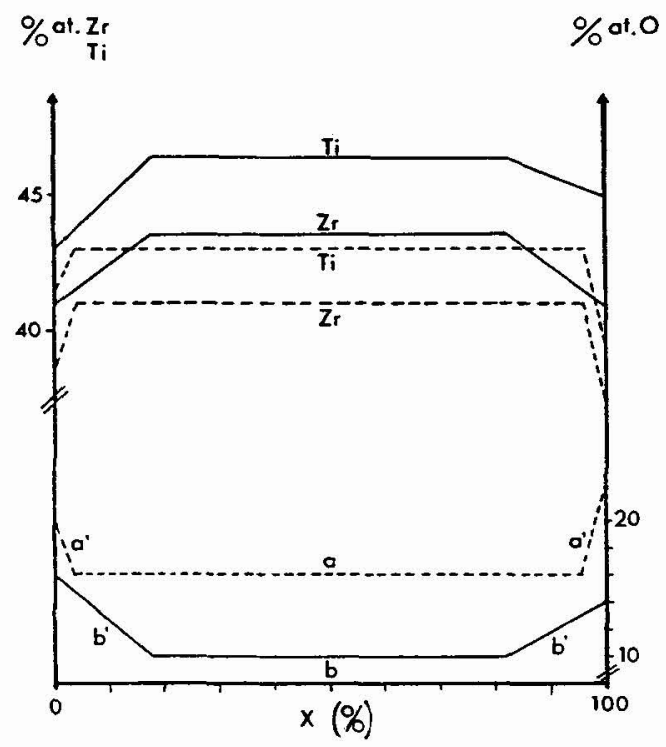

Fig. 3. - Profils de concentration de titane, zirconium et oxygène en fonction de l'épaisseur $x$ (ramenée à 100) pour : (- - ) la zone étroite $\mathrm{A}$; (-) la zone large $\mathrm{B}$.

a) Les compositions relatives du titane et du zirconium (c'est-à-dire en omettant la quantité d'oxygène) sont semblables dans les deux zones et proches de celles de départ (c'est-à- 
dire $47,5 \%$ atomique en zirconium et $52,5 \%$ atomique en titane). Il n'y a donc pas ségrégation d'une espèce métallique par rapport à l'autre dans le métal encore non oxydé, sous l'interface ;

b) Lépaisseur où l'on observe une décroissance de l'oxygène (Fig. 3, a') est presque inexistante dans la zone étroite, alors que dans la zone large elle s'étale sur une distance d'environ $100 \mu \mathrm{m}$ (Fig. 3, b').

Toutes ces constatations nous permettent de dire que la zone étroite A est formée d'une seule partie : celle-ci présente une dureté plus grande que celle de l'alliage initial, en raison de la grande quantité d'oxygène dissoute : $16 \%$ atomique (Fig. 3, a) ; elle sera qualifiée de dure. Dans la zone large $\mathrm{B}$, on distinguera par contre trois parties : deux notées $\mathrm{B} I$ semblables à la zone étroite A (Fig. 3, $\mathrm{b}^{\prime}$ ), en contact avec l'oxyde et une partie moins dure B2, ayant dissout moins d'oxygène : $10 \%$ atomique (Fig. 3, b), comprise entre les deux précédentes.

Tout se passe donc comme si, dans la zone non encore oxydée, existait une interface (représentée en pointillés, Fig. 2), parallèle à l'interface ondulée métal/oxyde, délimitant des domaines de duretés différentes ; elle disparaît au niveau des zones étroites.

En parallèle à ces mesures expérimentales, des calculs de diffusion montrent que pour avoir une concentration de $10 \%$ atomique d'oxygène au coeur de l'échantillon (Fig. 3, partie b) il faut que le coefficient de diffusion de l'oxygène dans l'alliage soit de l'ordre de $10^{-9} \mathrm{~cm}^{2} / \mathrm{s}$.

En raison d'un manque de données bibliographiques sur les alliages $\mathrm{Ti}-\mathrm{Zr}$, nous avons estimé que le coefficient de diffusion de l'oxygène dans l'alliage, à $720^{\circ} \mathrm{C}$, était le même que celui dans le titane et/ou le zirconium à cette même température, soit $10^{-9} \mathrm{~cm}^{2} / \mathrm{s}$ en phase $\beta$ et $10^{-11} \mathrm{~cm}^{2} / \mathrm{s}$ en phase $\alpha$ [4-12]. Ces valeurs nous permettent de supposer que le coeur de l'échantillon est sous forme $\beta$.

De plus, en phase $\beta$, les métaux dissolvent de moins grandes quantités d'oxygène qu'en phase $\alpha$, l'oxygène stabilisant celle-ci. Or, la même analyse, réalisée sur un échantillon oxydé à $500^{\circ} \mathrm{C}$, sous 100 mbar d'air, jusqu'à un avancement 0.5 , met en évidence une composition uniforme de $16 \%$ atomique d'oxygène dans la zone non encore oxydée. A cette température, l'alliage ne peut s'oxyder qu'en phase $\alpha$ (Fig. 1). Si seule cette phase intervenait à $720^{\circ} \mathrm{C}$, on devrait observer une concentration d'au moins $16 \%$ atomique en oxygène dans l'alliage, ce qui n'est pas le cas.

C'est la raison pour laquelle, l'intervention de la phase $\beta$, à $720^{\circ} \mathrm{C}$, au coeur de l'échantillon paraît seule expliquer la composition de $10 \%$ atomique en oxygène.

\section{Discussion et conclusion.}

L'étude porte sur l'alliage Ti-Zr titrant 47,5\% atomique de zirconium. L'oxydation de plaquettes conduit, dans certaines conditions de température et d'épaisseur des échantillons, à observer des ondulations de l'interface alliage/oxyde.

L'hypothèse d'une oxydation préférentielle aux joints de grains, souvent évoquée dans le cas de l'oxydation du zirconium [13] pourrait être avancée. Cependant, dans cette hypothèse, quelle que soit l'épaisseur initiale de l'échantillon, les ondulations devraient toujours être observées et devraient conserver sensiblement le même espacement (environ $50 \mu \mathrm{m}$ correspondant à la taille des grains), ce qui n'est pas le cas.

D'après toutes ces analyses, il semblerait que l'ondulation de l'interface soit l'image de l'accomodation des contraintes dont l'échantillon est le siège durant l'oxydation. Ces contraintes ont été plus intenses là où l'interface présente des creux et l'intensité variable de ces contraintes doit être reliée aux premiers instants de la réaction $(\xi=0,1-0,2)$. 
En effet, la première couche d'oxyde formée est noire, très adhérente au substrat (Fig. 4a). Pour relâcher les contraintes, cette couche d'oxyde se décolle par endroit, entraînant la formation de champignons blancs (oxyde stoechiométrique) [2, 14]. Au niveau de ces zones, l'oxygène pénètre plus facilement et le développement local de l'oxyde génère ponctuellement une contrainte plus forte (Fig. 4b). Le substrat, en réponse à cette contrainte, se déforme s'il peut facilement fluer. Dans ce cas, soumis à l'action de deux champignons voisins, il peut se former entre eux, une "bosse" (Fig. 4c). La couche d'oxyde, moins plastique, suit l'ondulation mais casse en de nombreux endroits (Fig. 4d).
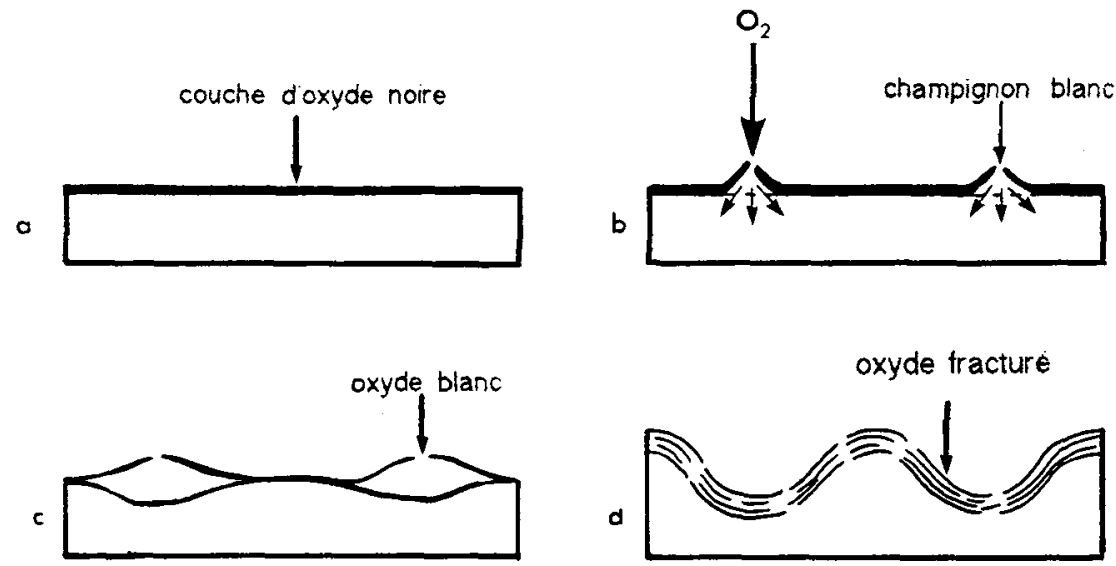

Fig. 4. - Mécanisme de génération de l'ondulation.

A la température considérée, la zone B2 (Fig. 2) de l'échantillon est sous forme $\beta$, alors que les parties $\mathrm{B} 1$ sont sous forme $\alpha$ en raison de l'effet alphagène de l'oxygène dissous [6].

La partie de l'alliage sous forme $\beta$ peut fluer au niveau de la limite $\alpha / \beta$ pour accomoder les contraintes. En effet, la phase $\beta$ possède de nombreux plans de glissement lui conférant une assez bonne plasticité ; la partie sous forme $\alpha$, d'épaisseur plus faible et adhérente à la phase $\beta$, suit le mouvement et transmet donc l'ondulation à l'interface alliage/oxyde. La couche d'oxyde, quant à elle, plus dure, ne peut se déformer et rompt perpendiculairement à l'interface en de nombreux endroits donnant à l'échantillon un aspect squameux.

Pour exister, les ondulations nécessitent simultanément 3 facteurs : a) une température critique dépendant de la concentration en zirconium : à basse température, la phase $\beta$ n'existant pas, le substrat sous forme $\alpha$ est peu plastique. Le fluage n'est donc pas possible et la résorption des contraintes se fait par rupture aux arêtes et stratification de l'oxyde.

A hautes températures, le fluage (dû à la phase $\beta$ ) intéresse la totalité de l'échantillon ; ce dernier présente alors un étirement au niveau des arêtes.

b) la présence de la phase $\beta$, liée à la température : seule cette phase, contenant de nombreux plans de glissement, peut fluer à température relativement basse et donc provoquer un déplacement de matière.

c) la dépendance des deux interfaces alliage/oxyde, liée à l'épaisseur initiale : si l'échantillon est très mince, la contrainte dûe à un champignon agit sur les deux interfaces en même temps, provoquant la courbure macroscopique de l'échantillon. Si par contre celui-ci est très épais, les contraintes sont résorbées dans la masse de la zone $\beta$ très importante. Seuls 
les échantillons moyennement épais ont des interfaces dépendantes et peuvent conduire à l'ondulation de l'interface.

On comprend aisément l'existence d'ondulations pour l'alliage $\operatorname{Ti}-\mathrm{Zr} 47,5 \%$ atomique de zirconium, puisqu'il suffit de choisir une épaisseur d'environ $1 \mathrm{~mm}$ pour réunir les trois conditions citées précédemment. En effet, la température de transition $\alpha / \beta$ étant très basse $\left(T=535^{\circ} \mathrm{C}\right)$, cet alliage peut, à des températures d'oxydation moyennes $\left(700^{\circ} \mathrm{C}\right.$ environ), être en grande partie sous forme $\beta$.

L'existence d'ondulations dans des alliages à plus forte teneur en zirconium pourrait être observée, mais cela nécessiterait des températures beaucoup plus élevées en raison d'une température de transition $\alpha / \beta$ déjà importante ; ceci a en effet été observé sur l'alliage Tỉ-Zr contenant $66,8 \%$ atomique de zirconium pour des températures supérieures à $700^{\circ} \mathrm{C}$.

\section{Bibliographie}

[1] GANNOT J.C., Thèse de Doctorat (Dijon, 1986).

[2] BENARD J., L'oxydation des métaux, T2 (Gauthier Villard, Paris, 1964).

[3] Pieraggi B., Dabosi F., J. Microsc. Spectrosc. Electron 5 (1980) 489.

[4] Pascal P., Nouveau Traité de Chimie Minérale, tome XX (Masson et Cie, Paris, 1960).

[5] Tomita M., TANABi T., Imoto S., Surf. Sci. 209 (1989) 173.

[6] Debuigne J., Guerlet J.P., Lehr P., IX Colloque de Métallurgie (Presse Universitaire Paris, 1966) p. 9.

[7] David D., ANSEl G., Boisot P., Beranger G., J. Electrochem. Soc. 122 (1975) 388.

[8] David D., Garcia E.A., LuCAS X., Beranger G., J. Less-Common Met. 65 (1979) 51.

[9] Mozhaev S.S., SokiRYANSki L.F., Titanium and its alloys (Kornilov, Moscou, 1965) p. 137

[10] RoE W.P., PALMER H.R., OPIE W.R., Trans. ASME 52 (1960) 191.

[11] DeChaMPS M., LeHR P., J. Less-Common Met. 56 (1977) 193.

[12] WASILEWSKI R.J., KeHL G.L, J. Inst. Met. 83 (1954) 94.

[13] GoDlewSKI J., Thèse de Doctorat (Compiègne, 1990).

[14] Belle J., MAllet M.V., J. Electrochem. Soc. 101 (1953) 339. 